\title{
Altered Expression of Nuclear Receptors Affects the Expression of Metabolic Enzymes and Transporters in a Rat Model of Cholestasis
}

\author{
Yoshitaka Hasegawa, ${ }^{a}$ Shuichi Kishimoto, ${ }^{*, a}$ Hirokazu Takahashi, ${ }^{a}$ Nobuo Inotsume, ${ }^{b}$ \\ Yoshikazu TAKeUCHI, ${ }^{a}$ and Shoji FuKUSHIMA ${ }^{a}$ \\ ${ }^{a}$ Laboratory of Clinical Pharmaceutics, Faculty of Pharmaceutical Sciences, Kobe Gakuin University; 1-1-3 Minatojima, \\ Chuo-ku, Kobe 650-8586, Japan: and ${ }^{b}$ Division of Clinical Pharmaceutics, Hokkaido Pharmaceutical University School \\ of Pharmacy; 7-1 Katsuraoka, Otaru 047-0264, Japan. \\ Received July 24, 2009; accepted September 12, 2009; published online September 18, 2009
}

\begin{abstract}
Hepatic metabolism is altered in some clinical conditions owing to the changes in the expression of metabolic enzymes and transporters. Therefore, we think that investigating the altered expression of metabolic enzymes and transporters is of particular significance to studies on drug disposition in some clinical conditions. We also believe that a simultaneous in vivo investigation of all factors affecting nuclear receptors and regulated genes is important to understand the relationship between nuclear receptors and their target genes. In this study, we induced cholestasis in rats by bile duct ligation (BDL), and investigated the changes in the mRNA expression of metabolic enzymes, transporters, and nuclear receptors and the protein levels of nuclear receptors in the nucleus by reverse transcriptase-polymerase chain reaction and Western blotting. In the liver of the rats subjected to BDL, the mRNA expression levels of cytochrome P450, conjugation enzymes, and transporters were concomitantly altered. The altered $\mathrm{mRNA}$ and protein levels of constitutive androstane receptor (CAR) and peroxisome proliferator-activated receptor $\alpha(\operatorname{PPAR} \alpha)$ in the nucleus were consistent with the changes in the plasma concentrations of total and conjugated bilirubin and fatty acid, respectively. The mRNA expression of CAR and PPAR $\alpha$ was linearly associated with the expression of the corresponding target genes. These results suggested that the increase in the levels of bilirubin and fatty acid on the BDL groups altered the mRNA and protein levels of CAR and PPAR $\alpha$, respectively in the nucleus, and this in turn altered the mRNA expression of metabolic enzymes and transporters as a hepatoprotective mechanism.
\end{abstract}

Key words bile duct ligation; nuclear receptor; transporter; cytochrome P450; metabolic enzyme

Pharmacological treatment has recently made a sizable contribution to medical care. Clarifying the mechanism of drug disposition is therefore desirable from the viewpoint of reducing the incidence of adverse drug reactions. Drug-metabolizing enzyme activity is markedly higher in the liver than in other organs. Because of the position of the liver, its weight, and its blood flow volume, many drugs are primarily metabolized here. Therefore, impairment of the drug metabolism in the liver, which occurs in hepatic disorders, has a considerable impact on drug disposition. ${ }^{1)}$

There have been several reports on altered hepatic metabolism in some clinical conditions such as hepatic disorders, kidney disorders, and diabetes mellitus, owing to the changes in the expression of metabolic enzymes and transporters. ${ }^{2-9}$ )

Hepatic metabolism of drugs is classified into phase 1, which is catalyzed by cytochrome P450 (CYP), and phase 2, which is catalyzed by enzymes such as uridine $5^{\prime}$-diphosphate (UDP)-glucuronosyltransferase (UGT), sulfotransferase (SULT), and glutathione $S$-transferase (GST). Drug metabolites produced in phase 1 and form polar compounds formed in phase 2 by conjugating with glucuronic acid, sulfuric acid, and glutathione. Consequently, the excretion of the drug in the urine or bile increases. Multidrug resistance protein (MDR), bile salt export pump (BSEP), multidrug resistanceassociated protein (MRP), organic anion transporter (OAT), organic cation transporter (OCT), and organic anion transporting polypeptide (OATP) have been identified as drug transporters in hepatocytes. ${ }^{10-14)}$ Therefore, investigations on the altered expression of metabolic enzymes and transporters are of particular significance to studies on drug disposition in some clinical conditions.
Nuclear receptors are elements that control the expression of metabolic enzymes and transporters. Nuclear receptors, which are normally present in the cytoplasm, are transported into the nucleus either directly by binding to ligands or indirectly by signal-mediated interactions. This is followed by heterodimerization between the nuclear receptors and the retinoid X receptor (RXR), which is normally present in the nucleus. These heterodimers bind to specific gene promoters and alter the expression of the target genes by interfering with transcription. ${ }^{14-17)}$ The pregnane X receptor (PXR), constitutive androstane receptor (CAR), farnesoid $\mathrm{X}$ receptor (FXR), and peroxisome proliferator-activated receptor (PPAR) have been identified as representative nuclear receptors in hepatocytes. ${ }^{18,19)}$ It is reported that (1) dexamethasone-induced expression of PXR modifies the expression of CYP3A, SULT2A1, MDR1a, MRP2, and OATP2; (2) phenobarbital-induced expression of CAR modifies the expression of CYP2B, UGT1A1, and MRP3; and (3) clofibrate-induced expression of PPAR $\alpha$ modifies the expression of CYP4A, UGT2B1, and MDR2. ${ }^{20-27)}$

There have been several reports on the altered expression of metabolic enzymes and transporters in rat models of cholestasis induced by bile duct ligation (BDL). Bohan et al. demonstrated that the expression of MRP2 decreases, that of MRP3 increases, and that of BSEP remains unchanged. ${ }^{28)}$ Dumont et al. reported that the expression of OATP1 and Nataurocholate cotransporting polypeptide (NTCP) reduces. ${ }^{29)}$ However, these studies only described individual nuclear receptors and not the association between them. Stahl et al. reviewed these studies on individual nuclear receptors and illustrated the network of nuclear receptors and regulated 
genes in cholestasis. ${ }^{30)}$ However, it is insufficient to only compile the results of individual studies because these networks are so complex. For diseases in particular, rather than accumulate the results of studies conducted on the cell level, integrated evaluation of the alterations occurring at the physiological level is necessary, because various factors may associate with these changes. We believe that a simultaneous in vivo investigation of all factors affecting nuclear receptors and regulated genes is important to understand the relationship between nuclear receptors and their target genes. In this study, we evaluated the correlation between the expression of metabolic enzymes, transporters, and nuclear receptors such as PXR, CAR, and PPAR $\alpha$ in the rat model of cholestasis. The target genes studied were CYP1A1, CYP1A2, CYP2B1/2, CYP3A1, CYP3A2, CYP4A1, UGT1A1, UGT2B1, SULT2A1, BSEP, MDR1a, MDR2, MRP2, MRP3, NTCP, OATP1, and OATP2.

\section{MATERIALS AND METHODS}

Materials Sepasol RNA I Super and horseradish peroxidase-conjugated goat anti-mouse immunoglobulin $\mathrm{G}$ (IgG) were obtained from Nacalai Tesque Inc., Kyoto, Japan. Glycogen solution and the SuperScript III first-strand synthesis system for reverse transcriptase-polymerase chain reaction (RT-PCR) were obtained from Invitrogen Life Technologies Inc., Tokyo, Japan. The KOD Dash kit was obtained from Toyobo Inc., Osaka, Japan. Sense and antisense primers for BSEP, MRP2, UGT1A1, UGT2B1, and SULT2A1 were obtained from Sigma Inc., Kanagawa, Japan. Sense and antisense primers for CYP1A1, CYP1A2, CYP2B1/2, CYP3A1, CYP3A2, CYP4A1, MDR1a, MDR2, MRP3, NTCP, OATP1, OATP2, and glyceraldehyde 3-phosphate dehydrogenase (GAPDH) were obtained from Bex Inc., Tokyo, Japan. A nuclear/cytosol fractionation kit was obtained from BioVision Inc., California, U.S.A. The bicinchoninic acid (BCA) protein assay kit was obtained from Pierce Biotechnology Inc., Illinois, U.S.A. Antibodies against PXR (H-160), CAR (M150), PPAR $\alpha(\mathrm{N}-19)$, and lamin B (S-20), and horseradish peroxidase-conjugated donkey anti-goat $\mathrm{IgG}$ was obtained from Santa Cruz Biotechnology Inc., California, U.S.A. Horseradish peroxidase-conjugated donkey anti-rabbit IgG and the ECL plus Western blot detection system were obtained from Amersham Biosciences, New Jersey, U.S.A. All the other reagents used were of the highest available quality and molecular biology grade.

Animals Twelve male Donryu rats aged 6 weeks were purchased from Japan SLC Inc., Shizuoka, Japan, and housed for 1 week in order to exclude unusual animals. Some of the rats (BDL group) were anesthetized with pentobarbital. The abdomen was cut open, the bile duct was ligated at 2 sites, and the region between these sites was transected to create a gap. The other rats (control group) were subjected to a sham operation and bred for $5 \mathrm{~d}$ after the surgery.

Five days after the sham operation (control group), liver tissue and blood samples were collected and stored at $-80^{\circ} \mathrm{C}$ until analysis. After 1,3 , or $5 \mathrm{~d}$ from the BDL surgery (BDL1, BDL3, or BDL5 groups, respectively), liver tissue and blood samples were collected and stored at $-80^{\circ} \mathrm{C}$ until analysis. The liver tissue was stored in RNA-later to prevent the decomposition of total RNA by RNase. The blood samples were centrifuged at $12000 \mathrm{rpm}$ for $10 \mathrm{~min}$ to obtain plasma samples.

We dispatched the samples to FALCO Biosystems Ltd. for determining the plasma concentrations of total and conjugated bilirubin, aspartate aminotransferase (AST), and alanine aminotransferase (ALT).

Rats were handled in accordance with the guidelines of the Kobe Gakuin University for the care of laboratory animals. The protocol for this animal study was approved by the Animal Experimentation Ethics Committee of Kobe Gakuin University.

Analysis of mRNA Expression by Reverse Transcriptase-Polymerase Chain Reaction Total RNA was extracted from the rat liver tissue $(100 \mathrm{mg})$ by using Sepasol RNA I Super, chloroform, and isopropanol. cDNA was synthesized from total RNA $(2 \mu \mathrm{g})$ by performing RT-PCR on the SuperScript III first-strand synthesis system, using KOD Dash DNA polymerase. To a $24.5 \mu \mathrm{l}$ reaction mixture containing KOD Dash DNA polymerase, $0.5 \mu \mathrm{l}$ synthetic cDNA and the sense and antisense primers were added. Denaturation $\left(95^{\circ} \mathrm{C}, 15 \mathrm{~s}\right)$, annealing $\left(62,59,56,50^{\circ} \mathrm{C}, 30 \mathrm{~s}\right)$, and $\mathrm{ex}-$ tension $\left(70^{\circ} \mathrm{C}, 30 \mathrm{~s}\right)$ were performed for $23-35$ cycles by using the iCycler system (DM9110, Bio-Rad Laboratories, Inc., Tokyo, Japan).

Degenerate sense and antisense primers for PCR were designed and chemically synthesized on the basis of the nucleotide sequences of rat CYP1A1 (341 bp), CYP1A (334 bp), CYP2B1/2 (458 bp), CYP3A1 (329 bp), CYP3A2 (348 bp), CYP4A1 (344 bp), UGT1A1 (373 bp), UGT2B1 (255 bp), SULT2A1 (264 bp), BSEP (565 bp), MDR1a (351 bp), MDR2 (143 bp), MRP2 (421 bp), MRP3 (423 bp), NTCP (500 bp), OATP1 (302 bp), OATP2 (496 bp), PXR (741 bp), CAR (477 bp), PPAR $\alpha$ (594 bp), and GAPDH (576 bp). The primer sequences are shown in Table 1.

The PCR products were resolved by performing electrophoresis on $2 \%$ agarose gels for $50 \mathrm{~min}$. After that, the agarose gels were stained with ethidium bromide. The bands were visualized using an ultraviolet (UV) transilluminator (Atto Corporation, Tokyo, Japan), and specific bands were quantified by densitometric analysis.

The mRNA Expressions and the Protein Levels Analysis of the Nuclear Receptor in the Nucleus The liver tissue samples (approximately $300 \mathrm{mg}$ ) obtained from the rats were placed in $1 \mathrm{ml}$ of ice-cold phosphate buffered saline (PBS) and cut finely using scissors. After homogenization, the suspension was centrifuged at $3000 \mathrm{rpm}$ for $3 \mathrm{~min}$ at $4{ }^{\circ} \mathrm{C}$. The supernatant was removed, and nuclear extracts were obtained using the nuclear/cytosol fractionation kit. The protein levels in the nuclear extracts were quantified using the BCA protein assay kit.

Sample buffer $(0.1$ м Tris-HCl, 1.3 м 2-mercaptoethanol, and $0.1 \mathrm{M}$ sodium dodecyl sulfate [SDS]) was added to an equal volume of the nuclear extract containing 10 or $30 \mathrm{mg}$ protein. This solution was heated at $95^{\circ} \mathrm{C}$ for $3 \mathrm{~min}$, and then resolved by electrophoresis in $10 \%$ SDS polyacrylamide gel for $90 \mathrm{~min}$ at $200 \mathrm{~V}$. The gel was transferred onto a polyvinylidene difluoride (PVDF) membrane by applying a $90 \mathrm{~mA}$ current for $60 \mathrm{~min}$, using a semi-dry blotting system. The PVDF membrane was shaken in blocking solution $(3 \%$ skim milk and $0.1 \%$ Tween 20 in PBS) for $60 \mathrm{~min}$ and subsequently washed 6 times with $50 \mu \mathrm{l}$ of Tween-PBS. The pri- 
Table 1. Sense and Antisense Primers Used for RT-PCR

\begin{tabular}{|c|c|c|}
\hline Gene & Sense primer $\left(5^{\prime}-3^{\prime}\right)$ & Antisense primer $\left(3^{\prime}-5^{\prime}\right)$ \\
\hline CYP1A1 & CCA TGA CCA GGA ACT ATG GG & TCT GGT GAG CAT CCA GGA CA \\
\hline CYP1A2 & GCA GGT CAA CCA TGA TGA GAA & CGG CCG ATG TCT CGG CCA TCT \\
\hline CYP2B $1 / 2$ & TCA GGA GGA AGC CCA ATG TT & CAG CAA AGA AGA GAG AGA GC \\
\hline CYP3A1 & GGA AAT TCG ATG TGG AGT GC & AGG TTT GCC TTT CTC TTG CC \\
\hline CYP3A2 & TAC TAC AAG GGC TTA GGG AG & CTT GCC TGT CTC CGC CTC TT \\
\hline CYP4A1 & GGT GAC AAA GAA CTA CAG C & AGA GGA GTC TTG ACC TGC CAG \\
\hline UGT1A1 & TGG TGTGCC GGA GCT CAT GTT C & AGA CAG CAG CAT ACT GGA GTC CC \\
\hline UGT2B1 & TGT TGG TAT TCC CTT GTT TGC & GTG CTT GGC TCC TTT GTG ACG \\
\hline SULT2A1 & TCC TCA AAG GAT ATG TTC CG & CAG TTC CTT CTC CAT GAG AT \\
\hline BSEP & TGC TTA TGG GAG GCG TAT & GGG CTG ACA GCA AGA ATC \\
\hline MDR1a & GAT GGA ATT GAT AAT GTG GAC A & AAG GAT CAG GAA CAA TAA A \\
\hline MDR2 & AAG AAT TTG AAG TTG AGC TAA GTG A & TGG TTT CCA CAT CCA GCC TAT \\
\hline MRP2 & ATC CTC AGC TGC TGA AGT TG & CTG ATC TTG GAT GCC AGA AC \\
\hline MRP3 & TCA AAG AGG AGA TCG CAG AG & AGC ATG AGG ATG GGG GCC AG \\
\hline NTCP & ATG CCC TTC TCT GGC TTT CT & GCT CCA TGG TTC TGA TGG TT \\
\hline OATP1 & AAG CGA AGA AGC TGG AAA CA & CAC CAC AGG TCT GTG CAG TT \\
\hline OATP2 & TGC ACA CTT AGC ATT CTG GC & TGC ATG TAA CCC AAC TCC AA \\
\hline PXR & GCC CAT ACG AAA CAC CAA AG & AGG GTC TTC CAA CAG TGA CG \\
\hline CAR & TCT CAC TCA ACA CTA CGT TC & CTG GGA AAG GAT CCA AGC CTG GG \\
\hline $\operatorname{PPAR} \alpha$ & CTG GTC AAG CTC AGG ACA CA & AAA CGG ATT GCA TTG TGT GA \\
\hline GAPDH & CCA TCA CCA TCT TCC AGG AG & CCT GCT TCA CCA CCT TCT TG \\
\hline
\end{tabular}

mary antibody diluted in Tween-PBS (dilution ratio: 500-fold for PXR and PPAR $\alpha, 300$-fold for CAR, and 2000-fold for lamin $\mathrm{B})(78 \mathrm{kDa})$ was added, and the membrane was incubated overnight at $4{ }^{\circ} \mathrm{C}$. On the next day, the membrane was washed 6 times with $50 \mu \mathrm{l}$ of Tween-PBS, and the secondary antibody diluted in Tween-PBS (dilution ratio: 10000-fold for PXR and CAR, 50000-fold for PPAR $\alpha$, 40000-fold for lamin B) was added. Subsequently, the membrane was shaken for $60 \mathrm{~min}$ at room temperature and washed 6 times with $50 \mu$ l of Tween-PBS.

The membrane was photographed using the ECL plus Western blotting detection system, and specific bands were quantified by densitometric analysis.

Statistical Analyses The data are presented as mean \pm standard deviation (S.D.). Parametric comparisons were made using analysis of variance (ANOVA). Significant difference was evaluated using Dunnett's test if ANOVA was significant. Values of $p<0.05$ were considered statistically significant.

\section{RESULTS}

Analysis of mRNA Expression by RT-PCR The concentrations of total and conjugated bilirubin, AST, and ALT in plasma were measured to determine if the BDL had been successful in inducing cholestasis associated with hepatopathy in the rats. The total bilirubin concentration was notably higher in the BDL groups (BDL1, $2.0 \pm 0.3 \mathrm{mg} \cdot \mathrm{dl}^{-1}$; BDL3, $3.6 \pm 0.2 \mathrm{mg} \cdot \mathrm{dl}^{-1}$; BDL5, 3.4 $\pm 0.1 \mathrm{mg} \cdot \mathrm{dl}^{-1}$ ) than in the control group $\left(0.1 \mathrm{mg} \cdot \mathrm{dl}^{-1}\right.$; the values for the $3 \mathrm{BDL}$ groups were the same). A similar trend was noted for the concentration of conjugated bilirubin. The plasma concentrations of AST and ALT were notably higher in the BDL1 group $\left(732.3 \pm 72.7 \mathrm{IU} \cdot \mathrm{L}^{-1}\right.$ and $308.7 \pm 55.3 \mathrm{IU} \cdot \mathrm{L}^{-1}$, respectively) than in the control group $\left(90.0 \pm 24.3 \mathrm{IU} \cdot \mathrm{L}^{-1}\right.$ and $39.7 \pm 6.0 \mathrm{IU} \cdot \mathrm{L}^{-1}$, respectively). However, these concentrations were lower in the BDL3 $\left(406.3 \pm 125.5 \mathrm{IU} \cdot \mathrm{L}^{-1}\right.$ and $106.7 \pm 18.0 \mathrm{IU} \cdot \mathrm{L}^{-1}$, respectively) and BDL5 $(591.0 \pm 31.3$
$\mathrm{IU} \cdot \mathrm{L}^{-1}$ and $151.0 \pm 12.3 \mathrm{IU} \cdot \mathrm{L}^{-1}$, respectively) groups than in the BDL1 group.

The mRNA expression of CYP1A1, CYP1A2, CYP2B1/2, CYP3A1, CYP3A2, and CYP4A1 in the BDL groups was investigated. The corresponding electrophoretic profiles and band strengths are shown in Fig. 1. The mRNA expression of CYP1A1 and CYP1A2 reduced significantly in the BDL groups after the surgery: by $36 \%$ and $56 \%$, respectively, in the BDL5 group as compared to the control group (Figs. $1 \mathrm{~B}, \mathrm{C})$. The mRNA expression of CYP2B1/2 increased with time after the surgery: the expression in the BDL5 group was approximately 3.6-fold higher than that in the control group (Fig. 1D). The mRNA expression of CYP3A1 and CYP3A2 in the BDL groups did not differ before and after the surgery (Figs. 1E, F). In the BDL1 group, the mRNA expression of CYP4A1 increased transiently to a level approximately 2.6fold higher than that in the control group and subsequently decreased with time (Fig. 1G).

In the BDL groups, the mRNA expression of UGT1A1, UGT2B1, and SULT2A1 was examined. The corresponding electrophoretic profiles and band strengths are shown in Fig. 2. The mRNA expression of UGT1A1 increased with time after the surgery: the expression in the BDL5 group was approximately 2.6-fold higher than that in the control group (Fig. 2B). The mRNA expression of UGT2B1 increased transiently in the BDL1 group, to a level approximately 1.7-fold higher than that in the control group (Fig. 2C). The mRNA expression of SULT2A1 in the BDL groups did not differ after the surgery (Fig. 2D).

Next, we examined the mRNA expression of canalicular transporters such as BSEP, MDR1a, MDR2, and MRP2 in the hepatocytes. The corresponding electrophoretic profiles and band strengths are shown in Fig. 3. The mRNA expression of BSEP, MDR1a, and MRP2 in the BDL groups did not differ before and after the surgery (Figs. 3B, C, E). The mRNA expression of MDR2 increased transiently in the BDL1 group, to a level approximately 3-fold higher than that in the control group (Fig. 3D). 



Fig. 1. Electrophoretic Profiles of the mRNA Expression of CYP in the Liver of Rats Subjected to BDL (A) and the Band Strengths of CYP1A1 (B), CYP1A2 (C), CYP2B1/2 (D), CYP3A1 (E), CYP3A2 (F), and CYP4A1 (G), Expressed as a Percentage of the Control Value

GAPDH was used as quantitative DNA. The values shown are mean \pm S.D. determined in an experiment performed in triplicate. $* p<0.05$, for the BDL groups compared with the control group.
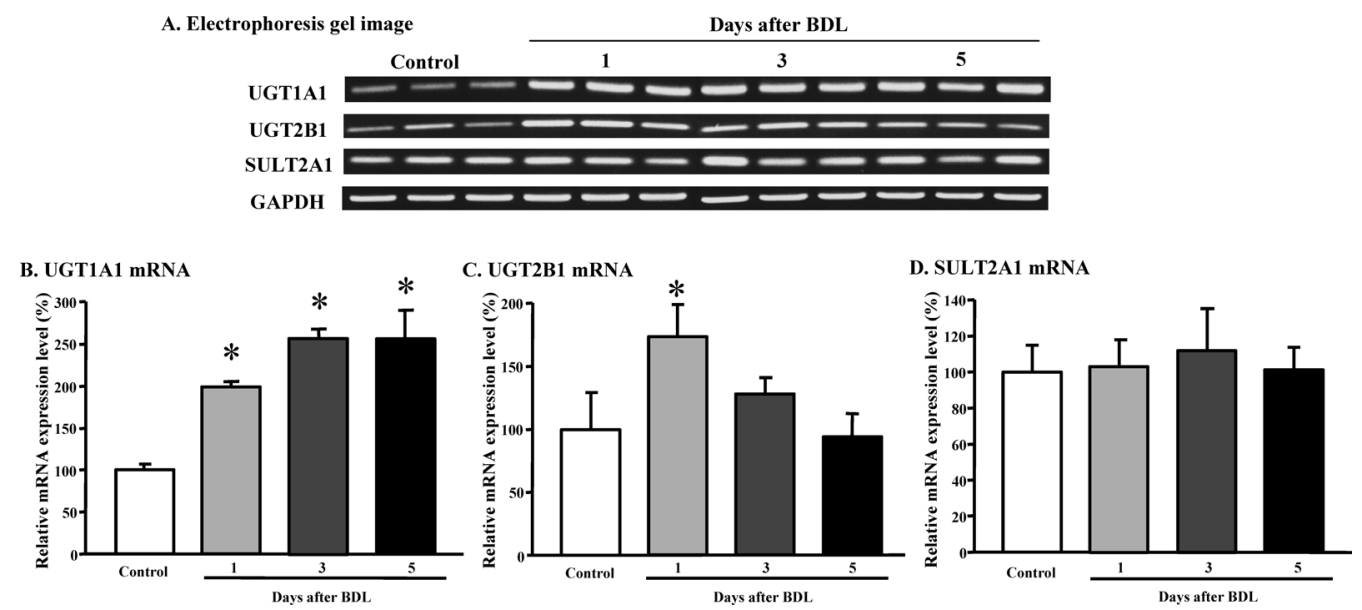

Fig. 2. Electrophoretic Profiles of the mRNA Expression of Conjugating Enzymes in the Liver of Rats Subjected to BDL (A) and the Band Strengths of UGT1A1 (B), UGT2B1 (C), and SULT2A1 (D), Expressed as a Percentage of the Control Value

GAPDH was used as quantitative DNA. The values shown are mean \pm S.D. determined in an experiment performed in triplicate. $* p<0.05$, for the BDL groups compared with the control group.

We also examined the mRNA expression of basolateral transporters such as MRP3, NTCP, OATP1, and OATP2 in the hepatocytes. The corresponding electrophoretic profiles and band strengths are shown in Fig. 4. The mRNA expression of OATP2 in the BDL groups did not differ after the surgery (Fig. 4E). The mRNA expression of MRP3 increased with time after the surgery: the expression in the BDL5 group was approximately 3.7 -fold higher than that in the control group (Fig. 4B). The mRNA expression of NTCP and OATP1 in the BDL groups significantly decreased after the surgery: this expression in the BDL5 group was approximately $60 \%$ that in the control group (Figs. 4C, D).

The mRNA Expressions and the Protein Levels Analysis of the Nuclear Receptor in the Nucleus We assessed the changes in the mRNA expression of the nuclear receptors PXR, CAR, and PPAR $\alpha$ in the liver of rats after BDL by per- 

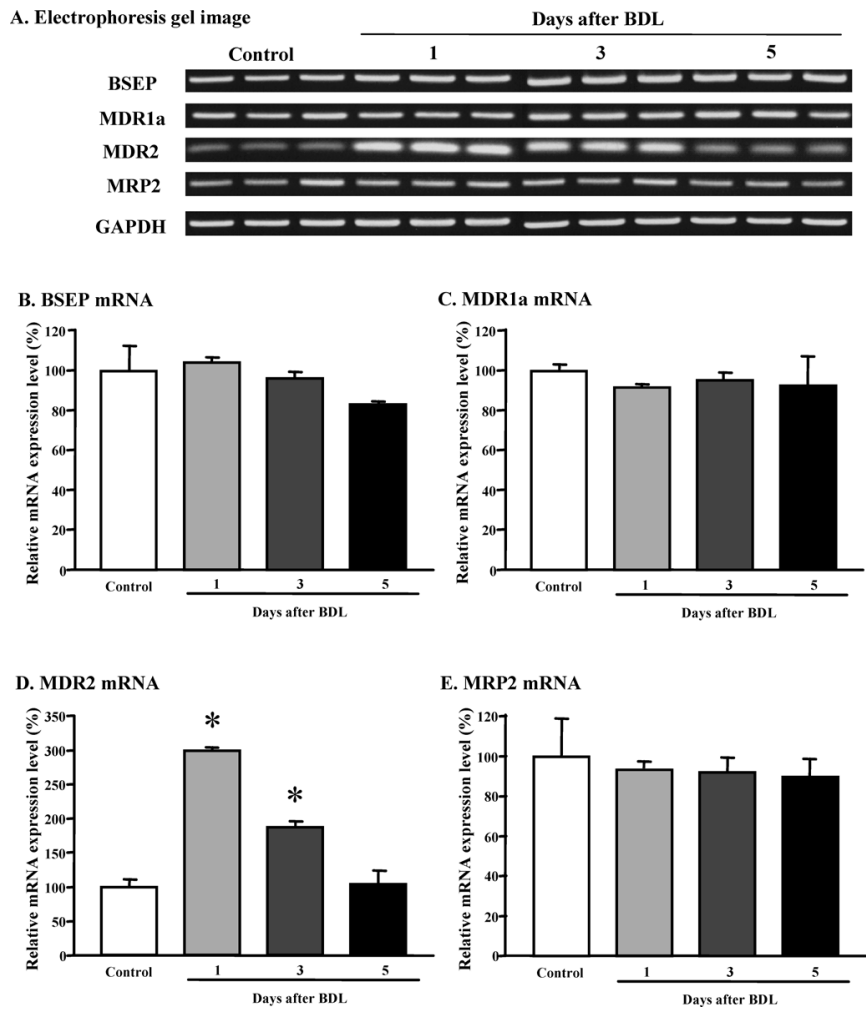

Fig. 3. Electrophoretic Profiles of the mRNA Expression of the Canalicular Transporter in the Liver of Rats Subjected to BDL (A) and the Band Strengths of BSEP (B), MDR1a (C), MDR2 (D), and MRP2 (E), Expressed as a Percentage of the Control Value

GAPDH was used as quantitative DNA. The values shown are mean \pm S.D. determined in an experiment performed in triplicate. $* p<0.05$, for the BDL groups compared with the control group.

forming RT-PCR as described above and the changes in the protein levels of these receptors in the nucleus by performing Western blotting. Lamin B was used as standard protein and mRNA. The corresponding electrophoretic profiles and band strengths are shown in Fig. 5.

In the BDL groups, the mRNA and the protein levels of PXR in the nucleus did not differ before and after the surgery (Fig. 5B). On the other hand, the levels of CAR notably increased after the surgery (Fig. 5C). In the BDL groups, the mRNA and the protein levels of $\operatorname{PPAR} \alpha$ in the nucleus increased transiently. In the BDL1 group, they increased to a level approximately 1.5 -fold higher than that in the control group and subsequently decreased with time (Fig. 5D).

\section{DISCUSSION}

In this study, we showed that the expression of CYP, conjugation enzymes, transporters, and nuclear receptors was concomitantly altered in the liver of rats with induced cholestasis. On the basis of these results, we discuss the correlation between the expression of nuclear receptors and target genes. The protein levels of nuclear receptors in the nucleus directly influence the expression of the target genes and alter the mRNA expression of nuclear receptors as a secondary effect. In this study, we showed that there exists a correlation between the mRNA and protein levels of nuclear receptors in the nucleus. Therefore, we compared the mRNA expression of nuclear receptors and the target genes.
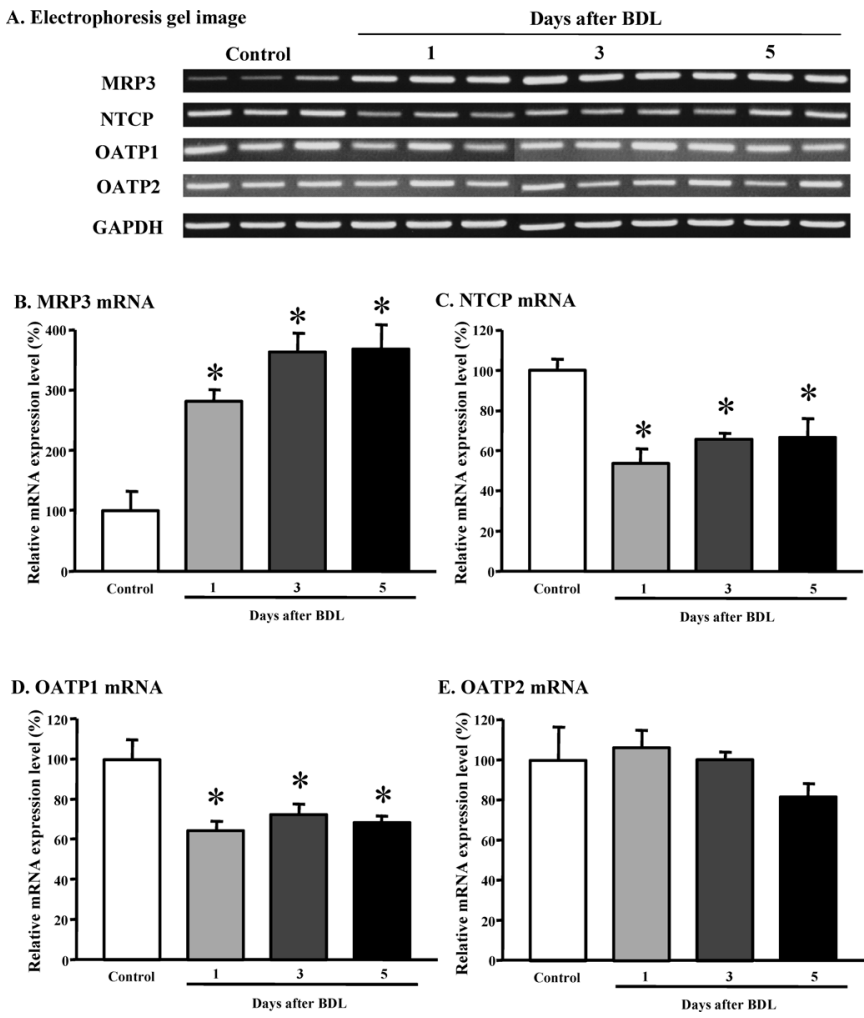

Fig. 4. Electrophoretic Profiles of the mRNA Expression of the Basolateral Transporter in the Liver of Rats Subjected to BDL (A) and the Band Strengths of MRP3 (B), NTCP (C), OATP1 (D), and OATP2 (E), Expressed as a Percentage of the Control Value

GAPDH was used as quantitative DNA. The values shown are mean \pm S.D. determined in an experiment performed in triplicate. $* p<0.05$, for the BDL groups compared with the control group.

Among genes of the CYP family, CYP1A, CYP2B, CYP3A, and CYP4A play a crucial role in drug metabolism. In humans, the CYP1A subfamily consists of 2 isozymes (CYP1A1 and CYP1A2) that are linked to the carcinogenesis of polycyclic aromatic hydrocarbons and allylamine. The CYP4A subfamily contains 13 isozymes. CYP4A11 in humans or CYP4A1 in rats is expressed in the liver and kidney, and cholesterol-lowering agents like clofibrate induce its expression. In addition, it is known that PPAR $\alpha$ contribute to the expression of CYP4A. ${ }^{31)}$ In this study, the alteration in the mRNA expression of CYP2B1/2 and CAR was quite similar. In addition, the alteration in the mRNA expression of CYP4A1 and PPAR $\alpha$ was also similar. These results indicate that the levels of CAR and PPAR $\alpha$ in the nuclear extract were associated with the expression of CYP2B1/2 and CYP4A1, respectively, in the liver of rats with induced cholestasis. In cholestasis, bile salts, bile acids, and fatty acids are accumulated in the liver, leading to hepatopathy. ${ }^{32}$ ) These compounds are also the major ligands of PXR, CAR, and PPAR $\alpha^{30}{ }^{30}$ Therefore, we hypothesized that in cholestasis, the levels of CAR and PPAR $\alpha$ increased to alter the expression of their target genes for detoxification. However, the main role of CYP3A is xenobiotic detoxification, and the mRNA expression of CYP3A1/2 did not change in cholestasis. In addition, the levels of the mRNA expression of CYP3A1/2 and PXR tended to be quite similar. In the cholestasis, the level of the substance, which usually excreted to bile, increases in the blood, and this might have influenced 

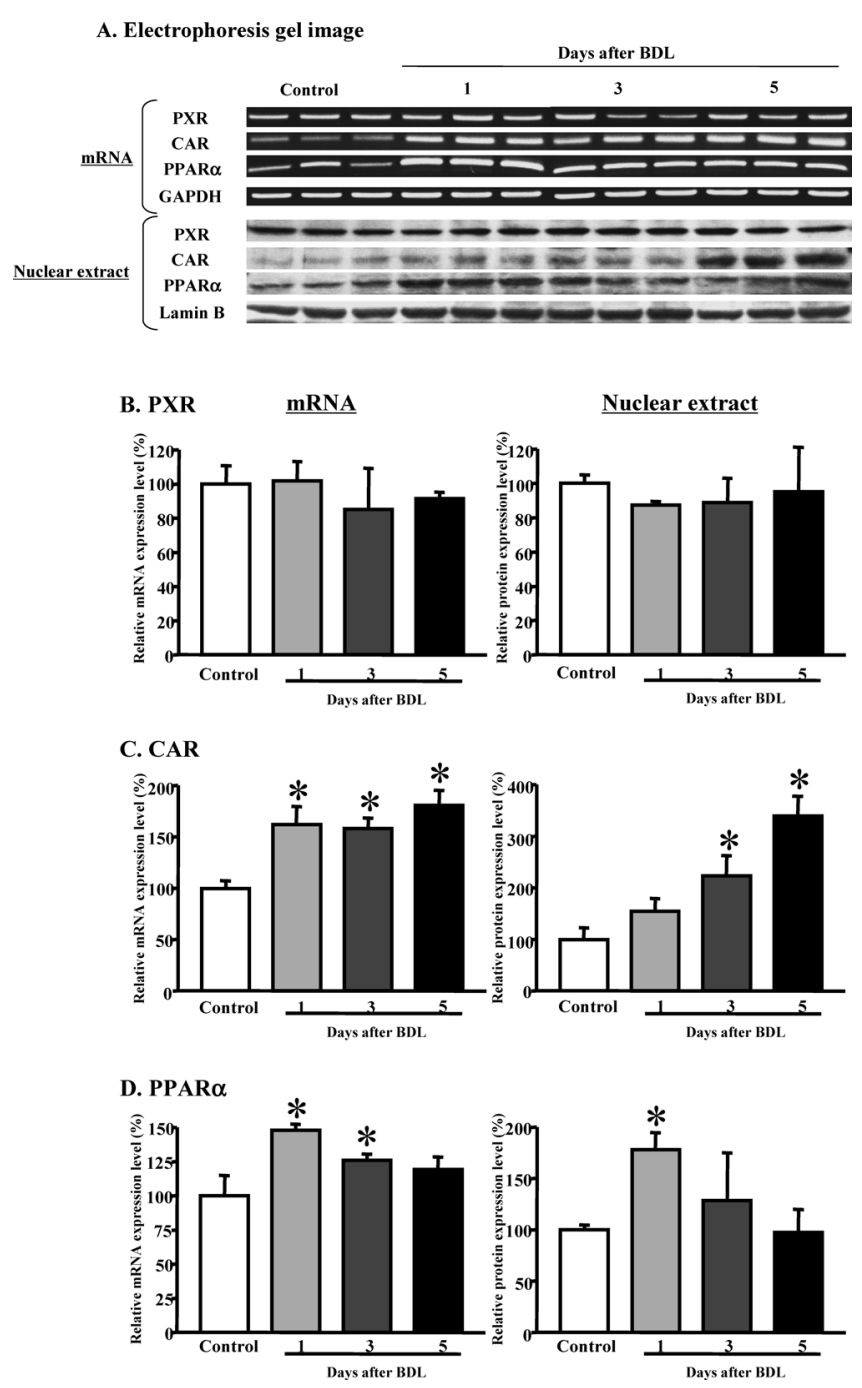

Fig. 5. Electrophoretic Profiles of the mRNA and the Protein Levels of Nuclear Receptors in the Nuclear Extract Obtained from the Liver of Rats Subjected to BDL (A) and the Band Strengths of PXR (B), CAR (C), and $\operatorname{PPAR} \alpha(\mathrm{D})$, Expressed as a Percentage of the Control Value

GAPDH was used as quantitative mRNA and lamin B was used as quantitative protein. The values shown are mean \pm S.D. determined in an experiment performed in triplicate. $* p<0.05$, for the BDL groups compared with the control group.

the expression of PXR because the expression of PXR is regulated by xenobiotic substances. It is difficult to mimic accurately this phenomenon in vitro because many xenobiotic substances complexify the expression of PXR in vivo. Thus, the expression of PXR shown in our study has the complexity, and we should clarify the alteration of the expression of nuclear receptors in disease states using animal models with comparing with the in vitro evidences.

Conjugation enzymes, especially UGT, SULT, and GST, play an important role in drug metabolism. Bilirubin, bile acids, thyroid hormones, and steroid hormones serve as a substrate for UGT, which catalyzes the glucuronidation reaction. Cholesterol, bile acids, and steroid hormones serve as a substrate for SULT, which catalyzes the reaction for displacement of the sulfate group. Halogen, epoxide, and nitro compounds provide a substrate for GST, which catalyzes the glutathione conjugation reaction. ${ }^{3,5,7,8)}$ It has been reported that (1) CAR alters the expression of UGT1A1, (2) PPAR $\alpha$ alters that of UGT2B1, and (3) PXR alters that of SULT2A1. ${ }^{25-27,33)}$
It has also been reported that CAR or the aryl hydrocarbon receptor (AhR) alters the expression of UGT1A1. ${ }^{22,34)}$ It is known that toxicants like bilirubin provide a substrate for UGT1A1 and UGT2B1. ${ }^{35)}$ In the present study, the expression of UGT1A1 and UGT2B1 increased in the BDL1 group. Hence, our results indicate that the increase in the bilirubin concentration in the BDL1 groups induced a transient increase in the expression of UGT with time. Although the expression of UGT1A1 increased with time, that of UGT2B1 transiently increased in the BDL1 group and subsequently decreased with time. These alterations were quite similar to that of CAR and PPAR $\alpha$, respectively. These results suggested that the expression of UGT2B1 in BDL3 and BDL5 was decreased because the expression of PPAR $\alpha$, which mediates the expression of UGT2B1, was decreased by other factors, i.e., bile salts, bile acids, and fatty acids. The mRNA expression of SULT2A1 and PXR did not change.

The uptake of drugs and their elimination via the liver involves a transporter-mediated pathway. The transporters in the liver are located on the canalicular and basolateral membranes; these include canalicular transporters such as MDR, MRP2, BSEP, and the breast cancer-resistance protein (BCRP) and basolateral transporters such as MRP3, OATP, and NTCP. All of these are important factors that influence drug disposition. ${ }^{36,37)}$ In this study, no changes were noted in the mRNA expression of BSEP, MDR1a, MRP2, and OATP2 in the BDL groups after the surgery and these alterations were quite similar to those of PXR. The mRNA expression of MDR2 increased transiently in the BDL1 group, while that of MRP3 increased with time after the surgery. We think that the cause of these alterations is the same as that of the alterations in UGT1A1 and UGT2B1.

In addition to the change in the above-mentioned transporters, the mRNA expression of NTCP and OATP1 significantly decreased in the BDL groups. Our results can be explained as follows. In cholestasis, toxicant accumulation due to decreased excretion of toxicants like bilirubin via MRP2 and bile acids via BSEP induces a compensatory increase in the expression of MRP3, which plays a role complementary to that of MRP2 and BSEP, i.e., protecting the liver by transporting toxicants into the blood. ${ }^{38)}$ For liver protection, the bile acid accumulated in the liver is contained within a micelle of phosphatidyl choline; therefore, MDR2 expression transiently increases to excrete the micelle. NTCP is involved in the uptake of bile components by the hepatocytes, and a decrease in its expression reduces this uptake.

The alteration in the mRNA expression of CAR and $\operatorname{PPAR} \alpha$ was linearly associated with that of the corresponding target genes in our rat model of cholestasis. These results suggest that the expression of CAR and PPAR $\alpha$ altered the expression of the corresponding target genes in this model. As stated above, the bilirubin, bile acid, and fatty acid levels in the liver were elevated in rats with cholestasis, and we consider that these elevated levels altered the mRNA and protein levels of both CAR and PPAR $\alpha$ in the nucleus, and this in turn altered the mRNA expression of CYP, metabolic enzymes, and transporters as a hepatoprotective mechanism.

From this study, it can explain the alteration of the drug disposition in the cholestasis as the change in the amount of ligand, the mRNA and protein levels of nuclear receptor in the nucleus, and the mRNA expression of CYP, metabolic 
enzymes, and transporters. In addition, it may forecast the alteration of the drug disposition in the cholestasis. Thus, we consider that useful information can be offered to the drug treatment in the cholestasis from the result of this study.

In this study, we showed that in our rat model of cholestasis, there exists a correlation between the mRNA and protein levels of nuclear receptors in the nucleus in vivo. On the other hand, we only examined the mRNA expression of metabolic enzymes and transporters. The correlativity of the mRNA expression and the protein level is not certain, though it is thought that these two tend to alter in the same manner. Therefore, it is necessary to clarify the networks between the expression of nuclear receptors and that of metabolic enzymes and regulated genes in the rat model of cholestasis in order to understand the protein levels of metabolic enzymes and transporters.

\section{REFERENCES}

1) Bastien M. C., Leblond F., Pichette V., Villeneuve J. P., Can. J. Physiol. Pharmacol., 78, 912-919 (2000).

2) Leblond F. A., Petrucci P. D., Dubé P., Bernier G., Bonnardeaux A., Pichette V., J. Am. Soc. Nephrol., 13, 1579-1585 (2002).

3) Maiti S., Grant S., Baker S. M., Karanth S., Pope C. N., Chen G., Biochem. Biophys. Res. Commun., 323, 235-241 (2004).

4) Pascussi J. M., Gerbal-Chaloin S., Drocourt L., Maurel P., Vilarem M. J., Biochim. Biophys. Acta, 1619, 243-253 (2003).

5) Shelby M. K., Cherrington N. J., Vansell N. R., Klaassen C. D., Drug Metab. Dispos., 31, 326-333 (2003).

6) Shimojo N., Int. J. Biochem., 26, 1261-1268 (1994)

7) Soars M. G., Fettes M., O'Sullivan A. C., Riley R. J., Ethell B. T., Burchell B., Biochem. Pharmacol., 65, 1251-1259 (2003).

8) 't Hoen P. A., Rooseboom M., Bijsterbosch M. K., van Berkel T. J., Vermeulen N. P., Commandeur J. N., Biochem. Pharmacol., 63, 1843 -1849 (2002).

9) Xu C., Li C. Y., Kong A. N., Arch. Pharm. Res., 28, 249-268 (2005).

10) Gottesman M. M., Ling V., FEBS Lett., 580, 998-1009 (2006).

11) Klaassen C. D., Slitt A. L., Curr. Drug Metab., 6, 309-328 (2005).

12) Maher J. M., Slitt A. L., Cherrington N. J., Cheng X., Klaassen C. D., Drug Metab. Dispos., 33, 956-962 (2005).

13) Sai Y., Drug Metab. Pharmacokinet., 20, 91—99 (2005).

14) van Meer G., Halter D., Sprong H., Somerharju P., Egmond M. R., FEBS Lett., 580, 1171-1177 (2006).

15) Kanno Y., Suzuki M., Nakahama T., Inoue Y., Biochim. Biophys. Acta, 1745, 215-222 (2005).
16) Makishima M., J. Pharmacol. Sci., 97, 177-183 (2005).

17) Xie W., Uppal H., Saini S. P., Mu Y., Little J. M., Radominska-Pandya A., Zemaitis M. A., Drug Discov. Today, 9, 442-449 (2004).

18) Eloranta J. J., Meier P. J., Kullak-Ublick G. A., Methods Enzymol., 400, 511-530 (2005).

19) Moore J. T., Moore L. B., Maglich J. M., Kliewer S. A., Biochim. Biophys. Acta, 1619, 235-238 (2003).

20) Barbier O., Duran-Sandoval D., Pineda-Torra I., Kosykh V., Fruchart J. C., Staels B., J. Biol. Chem., 278, 32852-32860 (2003).

21) Cherrington N. J., Slitt A. L., Maher J. M., Zhang X. X., Zhang J., Huang W., Wan Y. J., Moore D. D., Klaassen C. D., Drug Metab. Dispos., 31, 1315-1319 (2003).

22) Gardner-Stephen D., Heydel J. M., Goyal A., Lu Y., Xie W., Lindblom T., Mackenzie P., Randominska-Pandya A., Drug Metab. Dispos., 32, $340-347$ (2004).

23) Johnson D. R., Klaassen C. D., Toxicol. Sci., 67, 182-189 (2002).

24) Kitada H., Miyata M., Nakamura T., Tozawa A., Honma W., Shimada M., Nagata K., Sinal C. J., Guo G. L., Gonzalez F. J., Yamazoe Y., J. Biol. Chem., 278, 17838-17844 (2003).

25) Kullak-Ublick G. A., Stieger B., Meier P. J., Gastroenterology, 126, $322-342(2004)$

26) Sonoda J., Xie W., Rosenfeld J. M., Barwick J. L., Guzelian P. S., Evans R. M., Proc. Natl. Acad. Sci. U.S.A., 99, 13801-13806 (2002).

27) Zhou J., Zhang J., Xie W., Curr. Drug Metab., 6, 289-298 (2005).

28) Bohan A., Chen W. S., Denson L. A., Held M. A., Boyer J. L., J. Biol. Chem., 278, 36688-36698 (2003).

29) Dumont M., Jacquemin E., D’Hont C., Descout C., Cresteil D., Haouzi D., Desrochers M., Stieger B., Hadchouel M., Erlinger S., J. Hepatol., 27, 1051-1056 (1997).

30) Stahl S., Davies M. R., Cook D. I., Graham M. J., Xenobiotica, 38, 725-777 (2008).

31) Dickins M., Curr. Top. Med. Chem., 4, 1745-1766 (2004).

32) Tajuddin M., Tariq M., Bilgrami N. L., Kumar S., Adv. Myocardiol., 2, $209-212(1980)$

33) Sugatani J., Kojima H., Ueda A., Kakizaki S., Yoshinari K., Gong Q. H., Owens I. S., Negishi M., Sueyoshi T., Hepatology, 33, 1232-1238 (2001).

34) Yueh M. F., Haung Y. H., Hiller A., Chen S., Nguyen N., Tukey R. H., J. Biol. Chem., 278, 15001-15006 (2003).

35) Kren B. T., Parashar B., Bandyopadhyay P., Chowdhury N. R., Chowdhury J. R., Steer C. J., Proc. Natl. Acad. Sci. U.S.A., 96, 10349-10354 (1999).

36) Kruh G. D., Belinsky M. G., Oncogene, 22, 7537-7552 (2003).

37) Zollner G., Fickert P., Silbert D., Fuchsbichler A., Marschall H. U., Zatloukal K., Denk H., Trauner M., J. Hepatol., 38, 717-727 (2003).

38) van Waarde W. M., Verkade H. J., Wolters H., Havinga R., Baller J., Bloks V., Müller M., Sauer P. J., Kuipers F., Gastroenterology, 122, 1842-1852 (2002) 